\title{
Nonlinear state estimation and control for chaos suppression in MEMS resonator
}

\author{
Angelo Marcelo Tusset ${ }^{\mathrm{a}}$, Átila Madureira Bueno ${ }^{\mathrm{a}, *}$, Claudinor Bitencourt Nascimento ${ }^{\mathrm{a}}$, \\ Mauricio dos Santos Kaster ${ }^{\mathrm{a}}$ and José Manoel Balthazar ${ }^{\mathrm{b}}$ \\ ${ }^{a}$ Departamento de Engenharia Eletrônica, Universidade Tecnológica Federal do Paraná, Ponta Grossa, Paraná, \\ Brasil \\ ${ }^{\mathrm{b}}$ Departamento de Estatística, Matemática Aplicada e Computação, Instituto de Geociências e Ciências Exatas, \\ Rio Claro, Rio de Janeiro, Brasil
}

Received 3 October 2012

Revised 24 January 2013

Accepted 29 January 2013

\begin{abstract}
During the last decade the chaotic behavior in MEMS resonators have been reported in a number of works. Here, the chaotic behavior of a micro-mechanical resonator is suppressed. The aim is to control the system forcing it to an orbit of the analytical solution obtained by the multiple scales method. The State Dependent Riccati Equation (SDRE) and the Optimal Linear Feedback Control (OLFC) strategies are used for controlling the trajectory of the system. Additionally, the SDRE technique is used in the state estimator design. The state estimation and the control techniques proved to be effective in controlling the trajectory of the system. Additionally, the robustness of the control strategies are tested considering parametric errors and measurement noise in the control loop.
\end{abstract}

Keywords: Optimal control, SDRE control, chaos, MEMS, state estimation

\section{Introduction}

Recently, a significant research effort has been devoted to the report and to the analysis of the chaotic behavior in Microelectromechanical (MEM) resonators. Several works aim to provide design constraints for research, manufacturing or industrial applications of a simple Microelectromechanical System (MEMS), since many devices such as microsensors and micromachines can be built from MEMS. Nevertheless, in some cases, chaotic behavior is unavoidable appearing in the operation of MEMS, degrading the system's performance [1-4,8,27].

A number of works can be found in the litterature attempting to suppress the chaotic behavior in nonlinear dynamical systems by driving the system to a periodic orbit in the state space. The State Dependent Ricatti Equation (SDRE) and the Optimal Linear Feedback Control (OLFC) have been applied to chaos suppression in the following works $[6,10,11,19]$. Additionally, the SDRE and the OLFC techniques also have been applied to chaos suppression in MEMS [5,6]. Other techniques have also been applied in chaos suppression, such as the Robust Adaptive Fuzzy Control [8] and the Fuzzy Sliding Mode Control [9].

The SDRE techique [12-15] is an effective algorithm to synthesize nonlinear feedback control by allowing nonlinearities in the state space equations with design flexibility provided by state-dependent weighting matrices [16]. On

\footnotetext{
*Corresponding author: Átila Madureira Bueno, Departamento de Engenharia Eletrônica, Universidade Tecnológica Federal do Paraná, Av. Monteiro Lobato, km 4, s/n, Ponta Grossa, CEP: 84016-210, Paraná, Brasil. E-mail: atilabueno@utfpr.edu.br.
} 


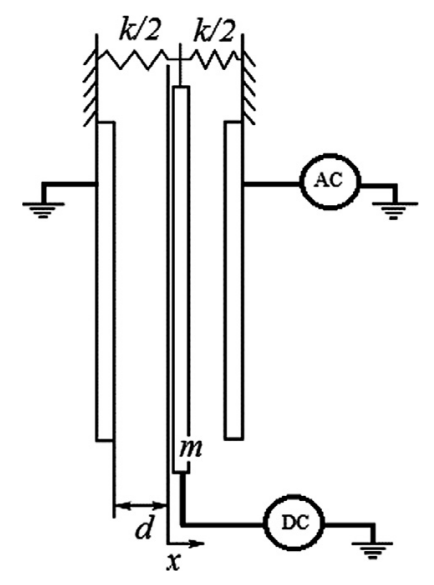

Fig. 1. Micro-mechanical resonator.

the other hand, the OLFC [17] considers the quadratic nonlinear Lyapunov function, explicitly expressing the form of a minimized functional in order to solve the optimal nonlinear control design problem, fulfilling the sufficient stability conditions $[18,19]$.

Here, the SDRE and OLFC techniques are used to suppress the chaotic behavior of a simple microelectromechanichal resonator. Additionally, a state feedback controller/observer is designed to suppress the chaotic behavior. The SDRE technique is used to allocate the poles of the state observer, and the observed states are used by the state feedback compensator in order to compute the control signal. In addition, the robustness of the control techniques are tested to parameter uncertainties, by means of a sensitivity analysis.

The paper is organized as follows. The mathematical model of the MEM resonator is shown in Section 2, with the Lyapunov exponents, Poincaré map and simulations results of the MEM resonator exhibiting chaotic behavior. In Section 3 an approximate analytical periodic solution is obtained by the perturbation method. The control system designs are shown in Section 4.

\section{Micro-mechanical resonator mathematical model}

The MEM resonator considered in this work is shown in Fig. 1 as consisting of a movable plate between two fixed plates (see [9]). The voltage $V(t)$ applied to the movable plate is composed of a polarization voltage $V_{p}$, and an alternating voltage $V_{i} \sin (\omega t)$.

In Fig. $1 d$ is the distance between the plates, $k$ is the conservative spring force coefficient, whereas (in Eq. (2)) $k_{1}$ and $k_{2}$ represente the linear and nonlinear spring force coefficients, $x$ is the lateral movement, $m$ is the front panel mass, $\mathrm{AC}$ is the polarization voltage $\left(V_{p}\right)$ and DC is the alternating voltage $\left(V_{i} \sin (\omega t)\right)$.

The equation of motion of the central plate is given by:

$$
m \ddot{x}=-F_{k}-F_{c}+F_{e}
$$

where $F_{k}$ is the conservative spring force, $F_{c}$ is the force due to damping effects and $F_{e}$ is the electric force.

The mechanical forces [6] are given by:

$$
\begin{aligned}
& F_{k}=k_{1} x+k_{3} x^{3} \\
& F_{c}=c \dot{x}
\end{aligned}
$$

and the electrical force [20] by:

$$
F_{e}=\frac{1}{2} \frac{C_{0}}{(d-x)^{2}}\left(V_{p}+V_{i} \sin (\omega t)\right)^{2}-\frac{1}{2} \frac{C_{0}}{(d+x)^{2}} V_{p}^{2}
$$


where $C_{0}$ is the capacitance of the parallel plates.

From the foregoing equations the MEM resonator equation of motion is given by:

$$
m \ddot{x}+c \dot{x}+k_{1} x+k_{3} x^{3}=\frac{1}{2} \frac{C_{0}}{(d-x)^{2}}\left(V_{p}+V_{i} \sin (\omega t)\right)^{2}-\frac{1}{2} \frac{C_{0}}{(d+x)^{2}} V_{p}^{2},
$$

and considering the following relations [8]: $w=\frac{\omega}{\omega_{0}}, t=\omega_{0} T, \omega_{0}^{2}=\frac{k_{1}}{m}, u=\frac{x}{d}, \mu=\frac{c}{m \omega_{0}}, \alpha_{3}=\frac{k_{3} d^{2}}{m \omega_{0}}, \gamma=\frac{C_{0} V_{p}^{2}}{2 m \omega_{0}^{2} d^{3}}$, and $\sigma=\frac{2 \gamma V_{i}}{V_{p}}$, the dimensionless equation of motion is given by:

$$
u^{\prime \prime}+\mu u^{\prime}+u+\alpha_{3} u^{3}=\gamma\left(\frac{1}{(1-u)^{2}}-\frac{1}{(1+u)^{2}}\right)+\frac{\sigma}{(1-u)^{2}} \sin (w T) .
$$

Defining the state variables as:

$$
\begin{aligned}
& y_{1}=u \\
& y_{2}=u^{\prime}
\end{aligned}
$$

the dimensionless equation of motion Eq. (6) is transformed into the state space equation:

$$
\begin{aligned}
& y_{1}^{\prime}=y_{2} \\
& y_{2}^{\prime}=-\mu y_{2}-y_{1}-\alpha_{3} y_{1}^{3}+\gamma\left(\frac{1}{\left(1-y_{1}\right)^{2}}-\frac{1}{\left(1+y_{1}\right)^{2}}\right)+\frac{\sigma}{\left(1-y_{1}\right)^{2}} \sin (w T)
\end{aligned}
$$

The system of Eq. (9) has been studied in [8,9], showing chaotic motion for the set of parameters $\mu=0.01$, $\alpha_{3}=12, \gamma=0.338, \sigma=0.03558$ and $w=0.5$, as it can be seen in Fig. 2. The plate displacement is shown in Fig. 2(a) and the phase portrait in Fig. 2(b). In Fig. 2(c) it can be seen that the system has a positive Lyapunov exponent. In Fig. 2(d) the Poincaré map is shown.

From Fig. 2 it can be seen that the MEM resonator system presents chaotic behavior. In order to suppress the chaotic motion the control system drives the system to a previously determined periodic orbit. That periodic orbit is determined by the perturbation method in the next section.

\section{Analytical solution}

In order to obtain the approximate analytical solution the multiple scales method is used as described in [21]. However, to simplify the mathematical reasoning, the rational terms in Eq. (9) are replaced by a polynomial function, around the equilibrium point. The techinique follows $[25,26]$ due to the fact that the van der Waals force is highly nonlinear, it is written in terms of the Taylor series expation, considering the constant, the linear, the cubic and the quadratic terms. This approach simplifies the mathematical reasoning, when applying the perturbation techniques, and the analysis of the damping and elastic constant parameters, since the cubic and quadratic terms are easier to handle. However,

Here, the polynomial approximation [22] minimizes the error functional

$$
J=\int_{-0.5}^{0.5}[f(u)-P(u)]^{2} d u
$$

resulting in the following approximations:

$$
\begin{aligned}
\frac{1}{(1-u)^{2}}-\frac{1}{(1+u)^{2}} & =11.0263 u^{3}+0.0674 u^{2} \\
\frac{1}{(1-u)^{2}} & =6.426 u^{3}+4.498 u^{2}+1.8525 u+0.9585 .
\end{aligned}
$$




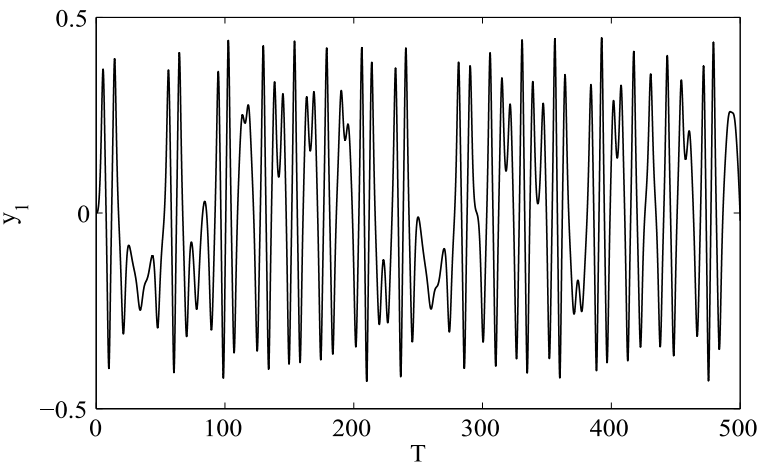

(a) MEM resonator displacement

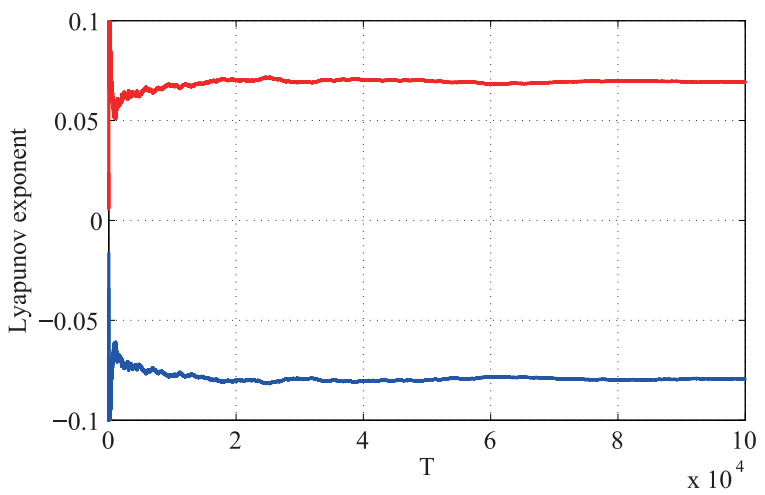

(c) Lyapunov exponents: $\lambda_{1}=0.0694$ and $\lambda_{2}=-0.0794$

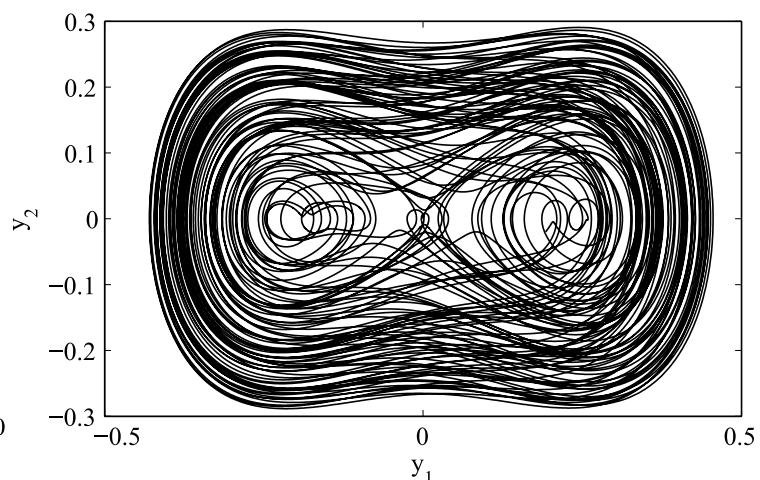

(b) Phase portrait

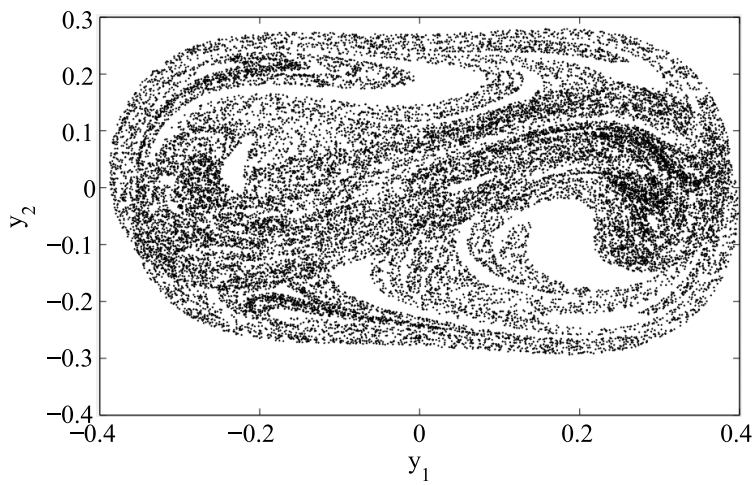

(d) Poincaré map

Fig. 2. Micromechanical resonator dynamics without control.

Replacing Eqs (11) and (12) into Eq. (6) results:

$$
u^{\prime \prime}+\mu u^{\prime}+u+a_{2} u^{2}+a_{3} u^{3}=a_{4} u^{3} \sin (w T)+a_{5} u^{2} \sin (w T)+a_{6} u \sin (w T)+f \sin (w T)
$$

where $a_{2}=-0.0228, a_{3}=8.2731, a_{4}=0.2286, a_{5}=0.16, a_{6}=0.0659, f=0.0341$ and $\mu=0.01$.

The multiple scales method is used to find an analytical solution of Eq. (13). This is done for a balance of order as follows $[6,7]$ :

$$
u^{\prime \prime}+u+\epsilon \mu u^{\prime}+\epsilon^{2} a_{2} u^{2}+\epsilon^{2} a_{3} u^{3}=\epsilon^{2} a_{4} u^{3} \sin (w T)+\epsilon^{2} a_{5} u^{2} \sin (w T)+\epsilon^{2} a_{6} u \sin (w T)+f \sin (w T)
$$

where $\epsilon$ is the parameter responsible for this balance [21]. Considering the scales $T_{0}=T$ and $T_{1}=\epsilon T$, the solutions are sought in the following way:

$$
u=u_{0}\left(T_{0}, T_{1}\right)+\epsilon u_{1}\left(T_{0}, T_{1}\right)+\cdots
$$

Since the independent variable $T$ was replaced by the scales $T_{0}$ and $T_{1}$, the derivatives with respect to $T$ should be expressed in terms of partial derivatives, such that:

$$
\begin{aligned}
\frac{d}{d T} & =D_{0}+\epsilon D_{1}+\cdots \\
\frac{d^{2}}{d T^{2}} & =D_{0}^{2}+2 \epsilon D_{0} D_{1}+\cdots
\end{aligned}
$$

Replacing Eq. (15) into Eq. (14) and considering the derivatives of Eq. (16) results:

$$
\begin{aligned}
& \left(D_{0}^{2}+2 \epsilon D_{0} D_{1}\right)\left(u_{0}+\epsilon u_{1}\right)+\epsilon \mu\left(D_{0}+\epsilon D_{1}\right)\left(u_{0}+\epsilon u_{1}\right)+\left(u_{0}+\epsilon \mu_{1}\right) \\
& \quad+\epsilon^{2} a_{2}\left(u_{0}+\epsilon u_{1}\right)^{2}+\epsilon a_{3}\left(u_{0}+\epsilon u_{1}\right)^{3}-\epsilon^{2} a_{4}\left(u_{0}+\epsilon u_{1}\right)^{3} \sin \left(w T_{0}\right) \\
& \quad-\epsilon^{2} a_{5}\left(u_{0}+\epsilon u_{1}\right)^{2} \sin \left(w T_{0}\right)-\epsilon^{2} a_{6}\left(u_{0}+\epsilon u_{1}\right) \sin \left(w T_{0}\right)-f \sin \left(w T_{0}\right)=0
\end{aligned}
$$


Since a second-order periodical solution is wanted, only the terms with $\epsilon^{0}$ and $\epsilon^{1}$ are extracted [7], yielding:

$$
\begin{aligned}
& \epsilon^{0}: D_{0}^{2} u_{0}+u_{0}=f \sin \left(w T_{0}\right) \\
& \epsilon^{1}: D_{0}^{2} u_{1}+u_{1}=-2 D_{0} D_{1} u_{0}-\mu D_{0} u_{0}-a_{3} u_{0}^{3} .
\end{aligned}
$$

A possible solution for Eq. (18) in polar form is:

$$
u_{0}=A\left(T_{1}\right) e^{i T_{0}}+\Lambda e^{i w T_{0}}+c c
$$

where

$$
\begin{aligned}
& A=\frac{1}{2} a e^{i \beta} \\
& \Lambda=\frac{f}{2\left(1-w^{2}\right)} .
\end{aligned}
$$

Replacing Eq. (20) into Eq. (19) results:

$$
\begin{aligned}
D_{0}^{2} u_{1}+a u_{1}= & -i\left(2 A^{\prime}+\mu A\right) e^{i T_{0}}+i\left(2 \bar{A}^{\prime}+\mu \bar{A}\right) e^{-i T_{0}}-i \mu w e^{i w T_{0}}+i \mu w e^{-i w T_{0}} \\
& -a_{3}\left[A\left(T_{1}\right) e^{i T_{0}}+\Lambda e^{i w T_{0}}+\bar{A}\left(T_{1}\right) e^{-i T 0}+\bar{\Lambda} e^{-i w T_{0}}\right]^{3} .
\end{aligned}
$$

Eliminating the secular terms in Eq. (23) results:

$$
2 i A^{\prime}+\mu i A+3 a_{3}\left(A \bar{A}+2 A^{2}\right)=0 .
$$

Replacing Eqs (21) and (22) into Eq. (24) results:

$$
\begin{aligned}
& a^{\prime}=0 \\
& \beta^{\prime}=\frac{3 a_{3} a^{2}}{8}+\frac{3 a_{3} a^{2}}{2} \cos (2 \beta)
\end{aligned}
$$

where $\beta$ is computed numerically by integrating Eq. (25). A solution for $u$ is given by:

$$
u=a \cos (T+\beta)+\frac{4 f}{2} \sin (w T)
$$

where $a=0.2, \beta=1.8228, f=0.0341$ and $w=0.5$.

Equation (26) is the sought periodic orbit.

\section{Nonlinear control design}

In this section the control system is designed aiming to suppress the chaotic behavior observed in Section 2. This is accomplished by determining the control signal $U$ that carries the system $\mathbf{e}(t)$, from any initial state, to the final state:

$$
\mathbf{e}(\infty)=\mathbf{0}
$$

with

$$
\mathbf{e}=\mathbf{y}-\tilde{\mathbf{y}}=\left[\begin{array}{ll}
y_{1}-\tilde{y}_{1} & y_{2}-\tilde{y}_{2}
\end{array}\right]^{\mathbf{T}}
$$

where $\tilde{\mathbf{y}}(t)$ is the vector of the desired orbit, and $\mathbf{y}(t)$ is the state space representation of the system of Eq. (6). 


\subsection{Aplication of the OLFC technique}

In this section the state space model of the MEM resonator in Eq. (9) is considered with the control law $U$, and is described by:

$$
\begin{aligned}
& y_{1}^{\prime}=y_{2} \\
& y_{2}^{\prime}=-\mu y_{2}-y_{1}-\alpha_{3} y_{1}^{3}+\frac{4\left(\gamma+2 \sigma \sin (w T)+y_{1} \sigma \sin (w T)\right) y_{1}}{\left(1-y_{1}^{2}\right)^{2}}+\frac{\sigma}{\left(1-y_{1}^{2}\right)^{2}} \sin (w T)+U
\end{aligned}
$$

with

$$
U=\tilde{u}_{0}+u_{0 f}
$$

where $u_{0 f}$ is the feedback control and $\tilde{u}_{0}$ is the feedforward control.

Considering

$$
\tilde{u}_{0}=\tilde{y}_{2}^{\prime}+\mu \tilde{y}_{2}+\tilde{y}_{1}+\alpha_{3} \tilde{y}_{1}^{3}-\frac{4\left(\gamma+2 \sigma \sin (w T)+\tilde{y}_{1} \sigma \sin (w T)\right) \tilde{y}_{1}}{\left(1-\tilde{y}_{1}^{2}\right)^{2}}-\frac{\sigma}{\left(1-\tilde{y}_{1}^{2}\right)^{2}} \sin (w T)
$$

and replacing Eq. (31) into Eq. (30) and considering Eq. (28) results:

$$
\begin{aligned}
e_{1}^{\prime}= & e_{2} \\
e_{2}^{\prime}= & -\mu e_{2}-e_{1}-\alpha_{3}\left(e_{1}+\tilde{y}_{1}\right)^{3}+\alpha_{3} \tilde{y}_{1}^{3}+\frac{\left(4 \gamma+2 \sigma \sin (w T)+\left(e_{1}+\tilde{y}_{1}\right) \sigma \sin (w T)\right)\left(e_{1}+\tilde{y}_{1}\right)}{\left(1-\left(e_{1}+\tilde{y}_{1}\right)^{2}\right)^{2}} \\
& -\frac{\left(4 \gamma+2 \sigma \sin (w T)+y_{1} \sigma \sin (w T)\right) \tilde{y}_{1}}{\left(1-\tilde{y}_{1}^{2}\right)^{2}}+\frac{\sigma}{\left(1-\left(e_{1}+\tilde{y}_{1}\right)^{2}\right)^{2}} \sin (w T)-\frac{\sigma}{\left(1-\tilde{y}_{1}^{2}\right)^{2}} \sin (w T)+u_{o f} .
\end{aligned}
$$

The system of Eq. (32) can be written in the following way:

$$
\mathbf{e}^{\prime}=\mathbf{A e}+\mathbf{G}(\mathbf{e}, \tilde{\mathbf{y}})+\mathbf{B} u_{o f}
$$

where

$$
\begin{aligned}
& \mathbf{A}=\left[\begin{array}{cc}
0 & 1 \\
-1 & -\mu
\end{array}\right], \\
& \mathbf{B}=\left[\begin{array}{l}
0 \\
1
\end{array}\right],
\end{aligned}
$$

and

$$
\left.\mathbf{G}(\mathbf{e}, \tilde{\mathbf{y}})=\left[\begin{array}{c}
0 \\
-\alpha_{3}\left(y_{1}-\tilde{y}_{1}\right)^{3}+\alpha_{3} \tilde{y}_{1}^{3}+\frac{\left(\frac{4 \gamma}{\sigma}+\sin (w T)\left(2+\left(y_{1}-\tilde{y}_{1}\right)\right)\right) \sigma\left(y_{1}-\tilde{y}_{1}\right)}{\left(1-\left(y_{1}-\tilde{y}_{1}\right)^{2}\right)^{2}}- \\
\frac{4\left(\gamma+2 \sigma \sin (w T)+y_{1} \sigma \sin (w T)\right) \tilde{y}_{1}}{\left(1-\tilde{y}_{1}^{2}\right)^{2}}+\left(\frac{\sigma}{\left(1-\left(y_{1}-\tilde{y}_{1}\right)^{2}\right)^{2}}-\frac{\sigma}{\left(1-\tilde{y}_{1}^{2}\right)^{2}}\right) \sin (w T)
\end{array}\right)\right] .
$$

According to $[18,19]$, if there are matrices $\mathbf{Q}, \mathbf{R}$ and $\mathbf{P}$, such that:

$$
\tilde{\mathbf{Q}}=\mathbf{Q}-\mathbf{G}^{T}\left(\mathbf{e}, x^{*}\right) \mathbf{P}-\mathbf{P G}\left(\mathbf{e}, x^{*}\right)
$$


is positive definite, then the control signal $u_{o f}$ is optimal, minimizing the cost functional:

$$
J=\int_{0}^{\infty} \mathbf{e}^{T} \tilde{\mathbf{Q}} \mathbf{e}+\mathbf{u}_{o f}^{T} \mathbf{R} \mathbf{u}_{o f} d t
$$

and transfers the nonlinear system from any initial condition to the final state of Eq. (27). The matrices $\mathbf{Q}, \mathbf{R}$ and $\mathbf{P}$ are the error weighting matrix, the control signal weighting matrix and the Riccati solution matrix, respectively.

The control signal $u_{o f}$ is determined by the equation

$$
\mathbf{u}_{o f}=-\mathbf{R}^{1} \mathbf{B}^{T} \mathbf{P e}=-\mathbf{k e}
$$

where $\mathbf{P}$ is the solution of the algebraic Riccati equation

$$
\mathbf{P A}+\mathbf{A}^{T} \mathbf{P}-\mathbf{P B R}^{-1} \mathbf{B}^{T} \mathbf{P}+\mathbf{Q}=\mathbf{0} .
$$

Considering

$$
\begin{aligned}
& \mathbf{Q}=\left[\begin{array}{cc}
10^{2} & 0 \\
0 & 10^{2}
\end{array}\right], \\
& \mathbf{R}=\left[10^{-2}\right],
\end{aligned}
$$

and solving the $\mathrm{LQR}$ problem using Matlab ${ }^{\mathrm{TM}}$, results:

$$
\mathbf{u}_{o f}=[-99.005-100.975] \mathbf{e}
$$

The simulation result for the OLFC technique is shown in Fig. 3, comparing the controlled and the uncontrolled phase portraits (Fig. 3(a)). Additionally, the error function $\mathbf{e}$ and the quadratic error function $L(T)=\mathbf{e}^{T} \tilde{\mathbf{Q}} \mathbf{e}$, where $\tilde{\mathbf{Q}}$ is computed from Eq. (37), are shown in Figs 3(b) and (c), respectively.

It can be noticed from Fig. 3 that the OLFC effectively drives the MEM resonator to the periodic orbit obtained from the multiple scales method (Eq. (26)), suppressing the chaotic behavior.

\subsection{Aplication of the SDRE technique}

In this section the SDRE is applied to the problem of chaos suppression for the MEM resonator. The dynamic system of Eq. (29) rewritten in the following form:

$$
\mathbf{y}^{\prime}=\mathbf{A}(\mathbf{y})+\mathbf{B U}_{s}+\mathbf{F}(\mathbf{y}, T)
$$

where $\mathbf{y}=\left[\begin{array}{ll}y_{1} & y_{2}\end{array}\right]^{T}$ is the time dependent state vector, $\mathbf{y}^{\prime} \in \mathrm{R}^{2}$ is the states first order time derivatives vector, $\mathbf{U}_{s}=\mathbf{u}_{s f}+\tilde{\mathbf{u}}_{s}$ is the control signal, $\mathbf{u}_{s f}$ is the feedback control signal, $\tilde{\mathbf{u}}_{s}$ is the feedforward control signal, and

$$
\begin{aligned}
\mathbf{A}(\mathbf{y}) & =\left[\begin{array}{cc}
0 & 1 \\
-1-\alpha_{3} y_{1}^{2}+\frac{\left(4 \gamma+2 \sigma \sin (w T)+y_{1} \sigma \sin (w T)\right)}{\left(1-y_{1}^{2}\right)^{2}} & -\mu
\end{array}\right], \\
\mathbf{B} & =\left[\begin{array}{l}
0 \\
1
\end{array}\right] \\
\mathbf{F}(\mathbf{y}, T) & =\left[\begin{array}{c}
0 \\
\frac{\sigma \sin (w T)}{\left(1-y_{1}^{2}\right)^{2}}
\end{array}\right] .
\end{aligned}
$$




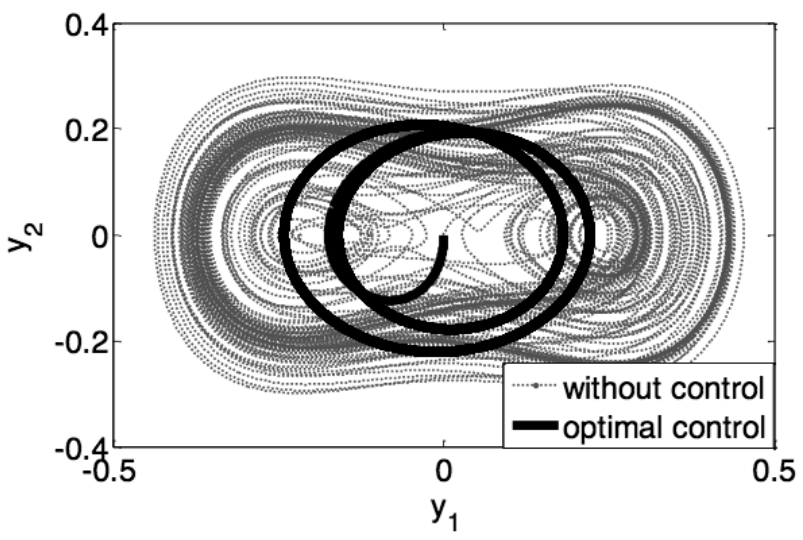

(a) Phase portrait comparing the chaotic orbit (uncontrolled) and the periodic (controlled) orbit

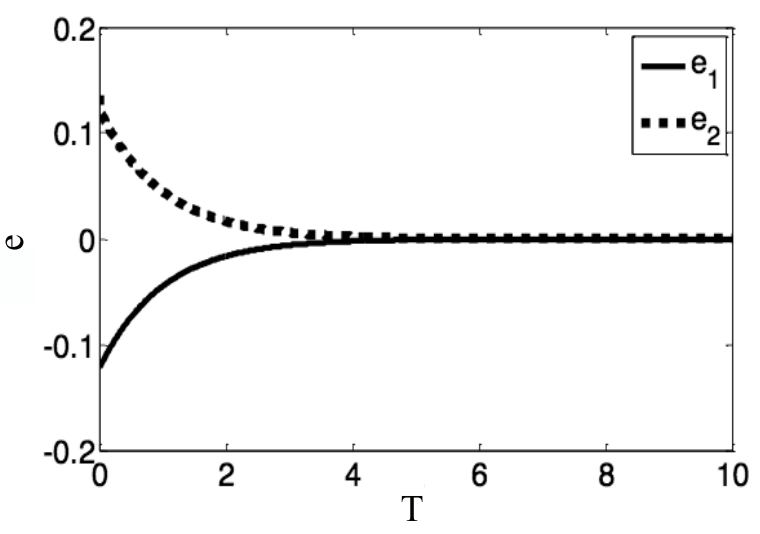

(b) Error function

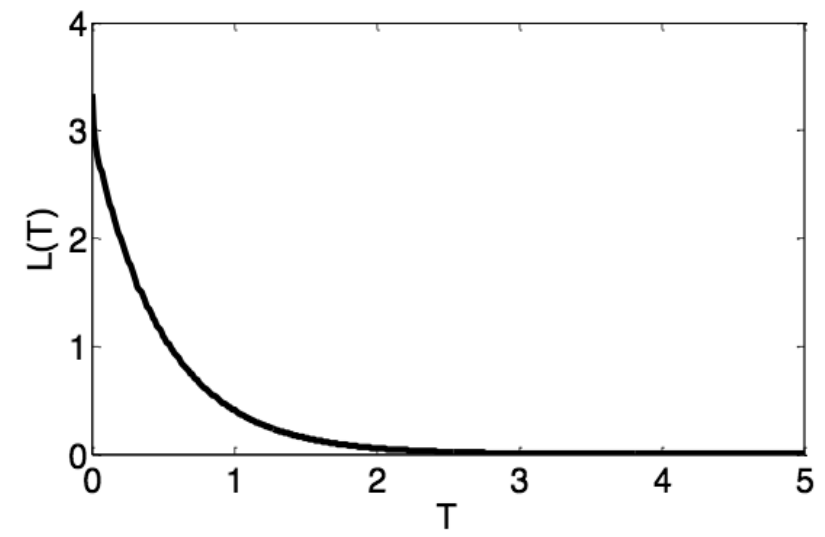

(c) Quadratic error function L(T)

Fig. 3. OLFC simulation results.

The cost function for the regulator problem is given by:

$$
J=\frac{1}{2} \int_{t_{0}}^{\infty} \mathbf{y}^{T} \tilde{\mathbf{Q}}(\mathbf{y}) \mathbf{y}+\mathbf{u}_{s f}^{T} \mathbf{R}(\mathbf{y}) \mathbf{u}_{s f} d t
$$

where $\mathbf{Q}(\mathbf{y})$ is a semi-positive definite matrix, and $\mathbf{R}(\mathbf{y})$ is positive definite.

Assuming full state feedback the control law is given by:

$$
\mathbf{u}_{s f}=-\mathbf{R}^{1}(\mathbf{y}) \mathbf{B}^{T}(\mathbf{y}) \mathbf{P}(\mathbf{y}) \mathbf{y}=-\mathbf{k}(\mathbf{y}) \mathbf{y},
$$

where $\mathbf{P}(\mathbf{y})$ is the solution of the SDRE, given by:

$$
\mathbf{A}^{T}(\mathbf{y}) \mathbf{P}(\mathbf{y})+\mathbf{P}(\mathbf{y}) \mathbf{A}(\mathbf{y})-\mathbf{P}(\mathbf{y}) \mathbf{B}(\mathbf{y}) \mathbf{R}^{-1}(\mathbf{y}) \mathbf{B}^{T}(\mathbf{y}) \mathbf{P}(\mathbf{y})+\mathbf{Q}(\mathbf{y})=\mathbf{0} .
$$

Defining the feedforward control signal as:

$$
\tilde{\mathbf{u}}_{s f}=\left[\begin{array}{c}
0 \\
-\frac{\sigma \sin (w T)}{\left(1-y_{1}^{2}\right)^{2}}
\end{array}\right],
$$

and replacing Eq. (51) into Eq. (44), results:

$$
\mathbf{y}^{\prime}=\mathbf{A}(\mathbf{y})+\mathbf{B} \mathbf{u}_{s f} .
$$




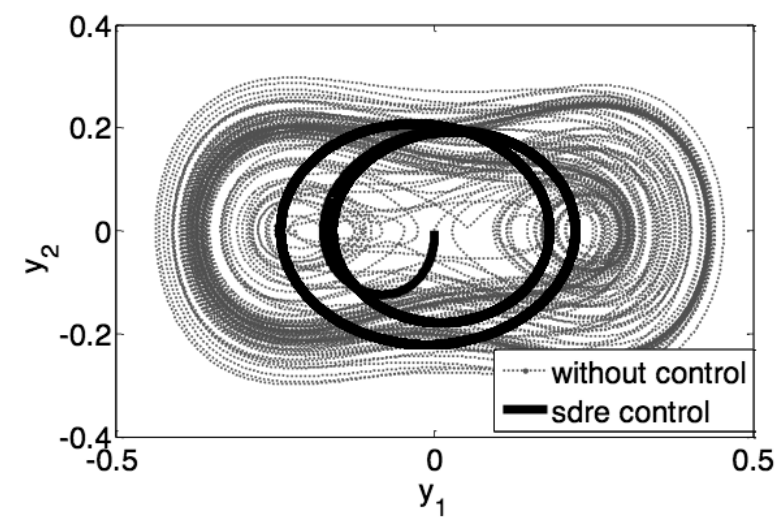

(a) Phase portrait comparing the chaotic orbit (uncontrolled) and the periodic (controlled) orbit

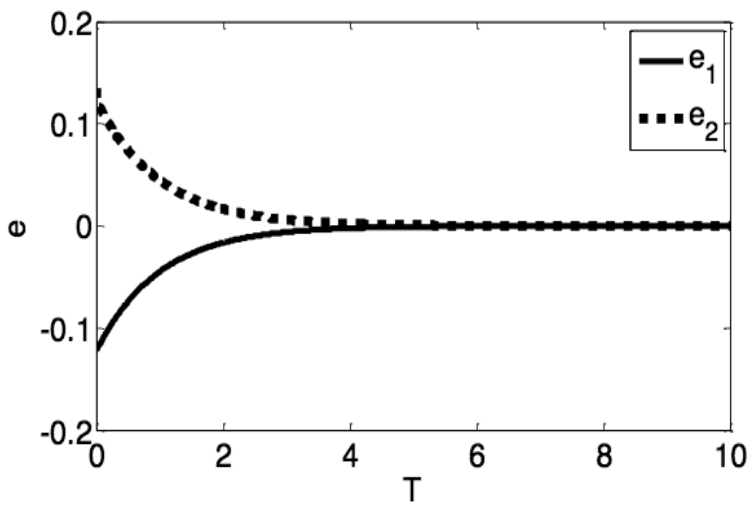

(b) Error function

Fig. 4. SDRE simulation results.

Choosing $\mathbf{Q}$ and $\mathbf{R}$ according to Eqs (41) and (42), and solving the LQR problem using Matlab ${ }^{\mathrm{TM}}$, the control signal $\mathbf{B u}_{s f}$ that drives the system to the desired periodic orbit can be computed.

The simulation results for the SDRE technique can be seen in Fig. 4. The SDRE technique also have been effective to suppress the chaotic behavior in the MEM resonator.

\subsection{Controler-observer design}

In this section a controller - observer is designed to suppress the chaotic behavior of the MEM resonator. Considering the system of Eq. (52) represented in the following form:

$$
\begin{aligned}
\mathbf{y}^{\prime} & =\mathbf{A}(\mathbf{y})+\mathbf{B} \hat{u} \\
z & =\mathbf{C y}
\end{aligned}
$$

Assuming that the state $\mathbf{y}$ can be approximated by the state $\hat{\mathbf{y}}$, such that

$$
\begin{aligned}
\hat{\mathbf{y}}^{\prime} & =\mathbf{A}(\hat{\mathbf{y}})+\mathbf{B} \hat{u}-\mathbf{K}_{0}(\hat{\mathbf{y}})(\mathbf{C y}-\mathbf{C} \hat{\mathbf{y}}) \\
\hat{z} & =\mathbf{C} \hat{\mathbf{y}}
\end{aligned}
$$

with

$$
\hat{u}=-\mathbf{R}^{1}(\hat{\mathbf{y}}) \mathbf{B}^{T}(\hat{\mathbf{y}}) \mathbf{P}(\hat{\mathbf{y}}) \hat{\mathbf{y}}=-\mathbf{k}(\hat{\mathbf{y}}) \hat{\mathbf{y}}
$$

where $\mathbf{P}(\mathbf{y})$ is the solution of the SDRE Eq. (50). Equation (55) represents the state observer. The last term of the right-hand side of Eq. (55) is a correction term involving the measured output and the estimated output signal.

Subtracting Eq. (53) from Eq. (55) yields the observer error, given by:

$$
e_{0}^{\prime}=\left(\mathbf{A}(\mathbf{y})-\mathbf{K}_{0}(\hat{\mathbf{y}}) C\right) \mathbf{y}-\left(\mathbf{A}(\hat{\mathbf{y}})-\mathbf{K}_{0}(\hat{\mathbf{y}}) \mathbf{C}\right) \hat{\mathbf{y}}
$$

where

$$
e_{0}^{\prime}=\mathbf{y}^{\prime}-\hat{\mathbf{y}}^{\prime}
$$

The dynamic behavior of the error vector is determined by the eigenvalues of the matrices:

$$
\begin{gathered}
\left(\mathbf{A}(\mathbf{y})-\mathbf{K}_{0}(\hat{\mathbf{y}}) C\right) \\
\left(\mathbf{A}(\hat{\mathbf{y}})-\mathbf{K}_{0}(\hat{\mathbf{y}}) C\right)
\end{gathered}
$$




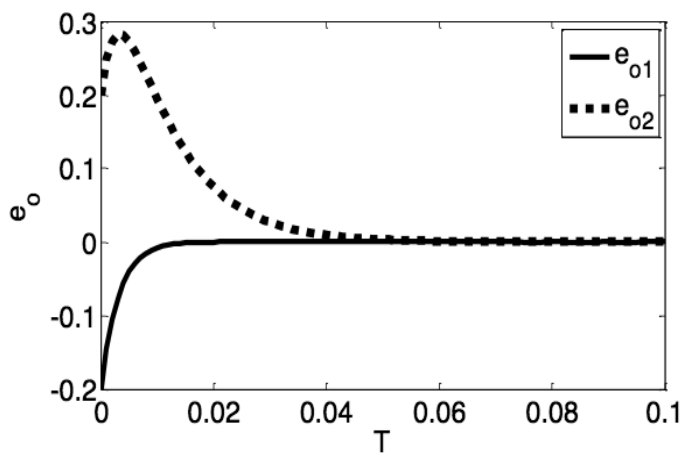

(a) Observer error

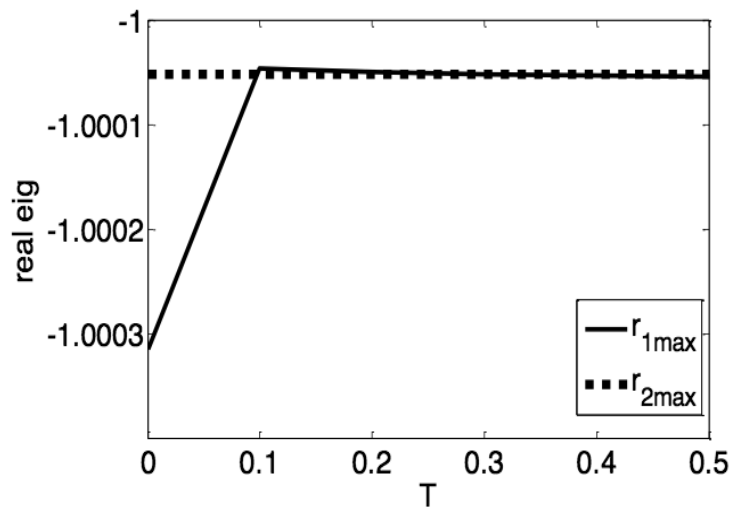

(c) Eigenvalues real part

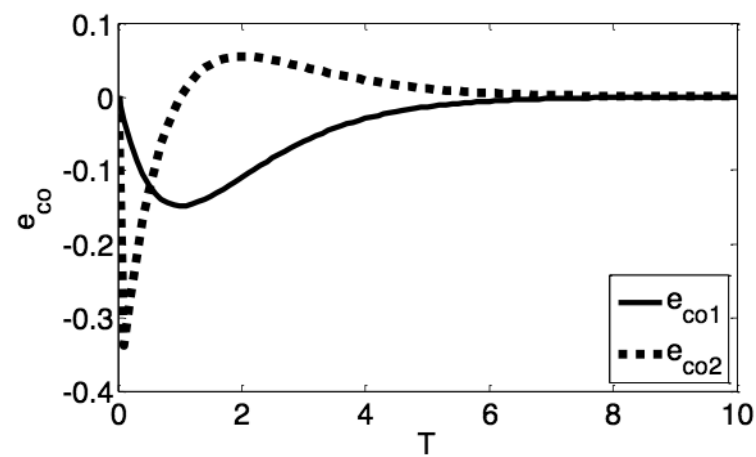

(b) Observer error

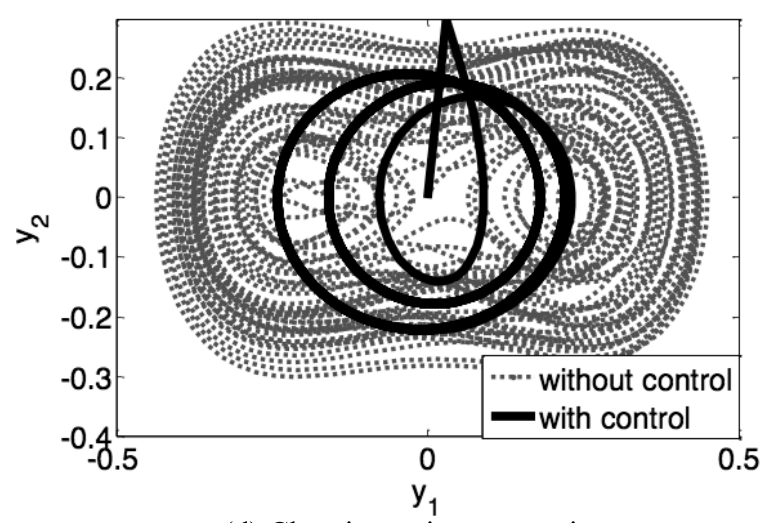

(d) Chaotic motion supression

Fig. 5. State observer controle simulation result.

If the eigenvalues of the matrices Eqs (60) and (61) have negative real part, then, for any initial error the estimation error will tend to zero over time. That is, $\hat{y} \rightarrow y$ for any $y(0)$ and $\hat{y}(0)$.

Therefore the choice of the weighting matrix $\mathbf{K}_{0}(\mathbf{y})$ must be such that the matrices Eqs (60) and (61) are stable. The matrix $\mathbf{K}_{0}(\mathbf{y})$ can be obtained by considering the optimal feedback gain [24] given by:

$$
\mathbf{K}_{0}(\hat{\mathbf{y}})=\hat{\mathbf{P}}(\hat{\mathbf{y}}) \mathbf{C}(\hat{\mathbf{y}}) \hat{\mathbf{R}}^{-1}(\hat{\mathbf{y}})
$$

where $\hat{\mathbf{P}}(\hat{\mathbf{y}})$ is the solution of the SDRE, given by:

$$
\hat{\mathbf{P}}(\hat{\mathbf{y}}) \mathbf{A}^{T}(\hat{\mathbf{y}})+\hat{\mathbf{A}}(\hat{\mathbf{y}}) \hat{\mathbf{P}}(\hat{\mathbf{y}})-\hat{\mathbf{P}}(\hat{\mathbf{y}}) \mathbf{C}^{T}(\hat{\mathbf{y}}) \mathbf{R}^{-1}(\hat{\mathbf{y}}) \mathbf{C}(\hat{\mathbf{y}}) \hat{\mathbf{P}}(\hat{\mathbf{y}})+\hat{\mathbf{Q}}(\hat{\mathbf{y}})=\mathbf{0}
$$

The cost function is given by:

$$
J=\frac{1}{2} \int_{t_{0}}^{\infty} \hat{\mathbf{y}}^{T} \hat{\mathbf{Q}}(\hat{\mathbf{y}}) \hat{\mathbf{y}}+\hat{\mathbf{u}}^{T} \hat{\mathbf{R}}(\hat{\mathbf{y}}) \hat{\mathbf{u}} d t
$$

where $\hat{\mathbf{Q}}(\hat{\mathbf{y}})$ is a semi-positive definite matrix, and $\hat{\mathbf{R}}(\hat{\mathbf{y}})$ is positive definite.

Considering

$$
\begin{aligned}
& \mathbf{Q}=\left[\begin{array}{cc}
10^{2} & 0 \\
0 & 10^{2}
\end{array}\right], \\
& \hat{\mathbf{Q}}=\left[\begin{array}{cc}
10^{3} & 0 \\
0 & 10^{3}
\end{array}\right], \\
& \mathbf{R}=\hat{\mathbf{R}}=\left[10^{-2}\right],
\end{aligned}
$$




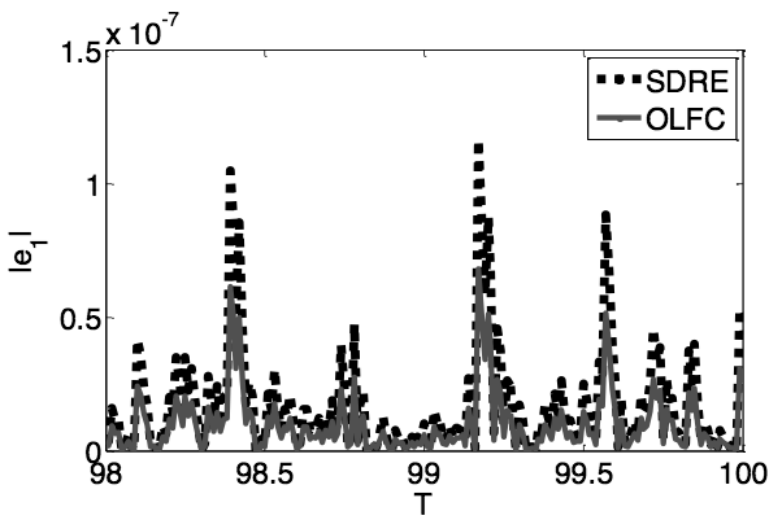

(a) $\left|e_{1}\right|$ without parameter uncertainties and noise measurement

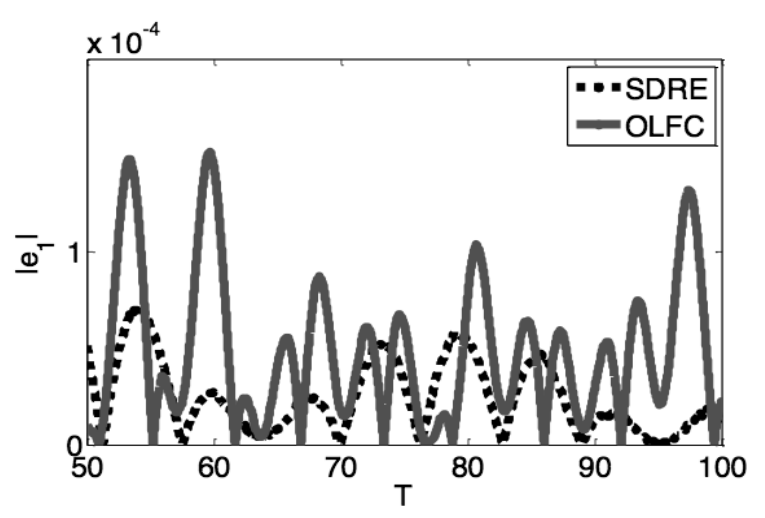

(c) $\left|e_{l}\right|$ with parameter uncertainties and noise measurement

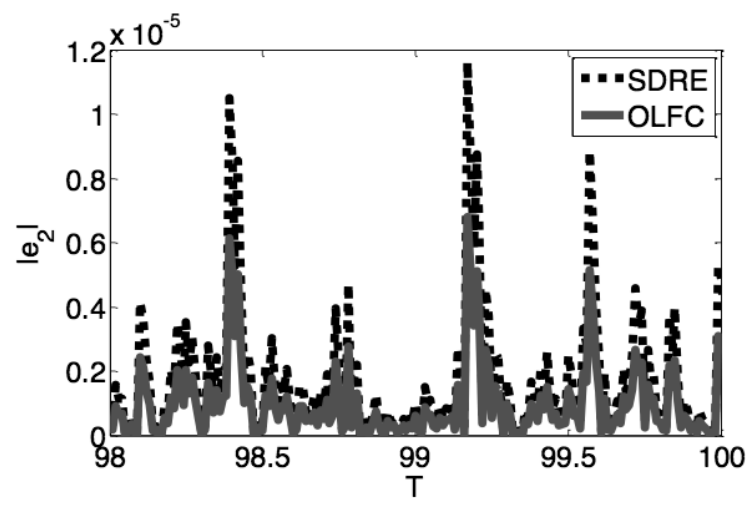

(b) $\left|e_{2}\right|$ without parameter uncertainties and noise measurement

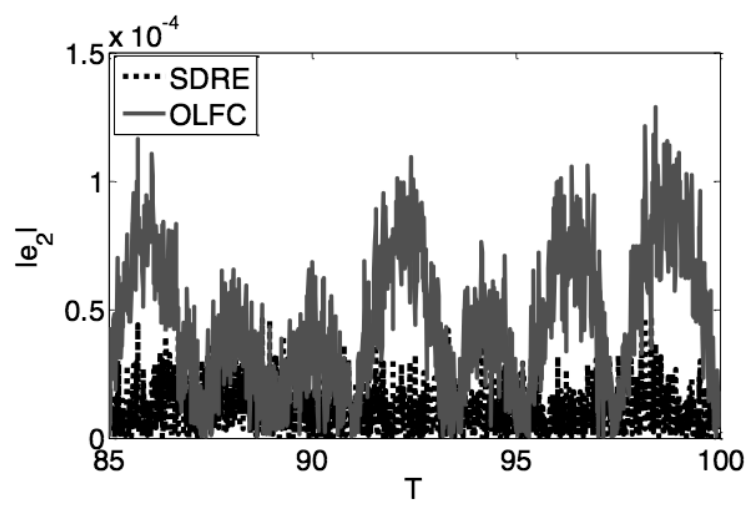

(d) $\left|e_{2}\right|$ with parameter uncertainties and noise measurement

Fig. 6. Comparing OLFC and SDRE in the presence of parameter uncertainties and noise measurement.

The state observer and the controller can be obtained. As it can be seen in Fig. 5 the proposed state observer for nonlinear systems is effective in suppressing the chaotic behavior in the MEM resonator. Additionally, using the SDRE to compute the matrix $\mathbf{K}_{0}(\hat{\mathbf{y}})$ generated stable matrices Eqs (60) and (61), resulting that the observer converge to the actual state.

\subsection{Control system performance in the presence of parametric errors and measurement noise}

Mathemematical models frequently present parametric uncertainties and are affected by measurement noise. In this section the performance OLFC, SDRE and state observer techniques are compared in the presence of parametric errors and measurement noise. The parameter uncertainties and the measurement errors are introduced in the system through a data set, as proposed by [23]. To test the effect of parameter uncertainties on the performance of the controller, the parameters in the control system will be added to a random function generating errors up to $\pm 20 \%$ of each parameter.

On the other hand, the effect of measurement noise on the performance of the controller is tested by adding to the control signal a sinusoidal function with random frequency and amplitude, given by:

$$
e_{\text {noise }}=\psi(T) \sin (\bar{\omega} T)
$$

where $\psi(T)=0.05 \pm 0.002 r(T), \bar{\omega}(T)=0.5 \pm 0.02 r(T)$, and $r(t)$ is a normally distributed random function. 


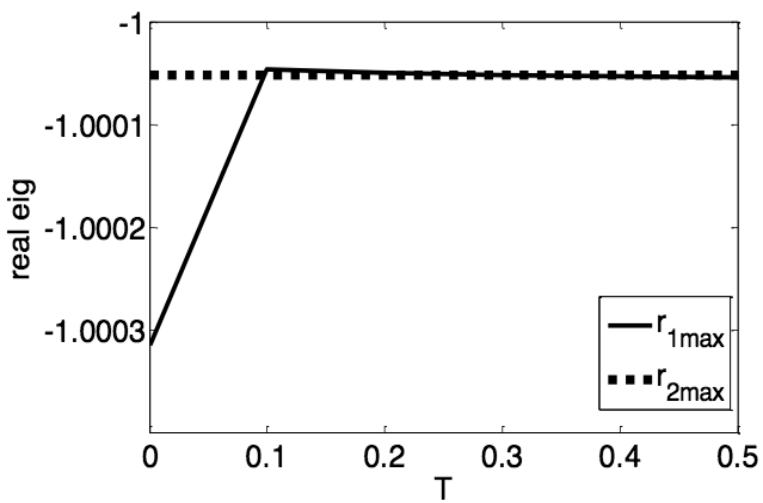

(a) Observer error

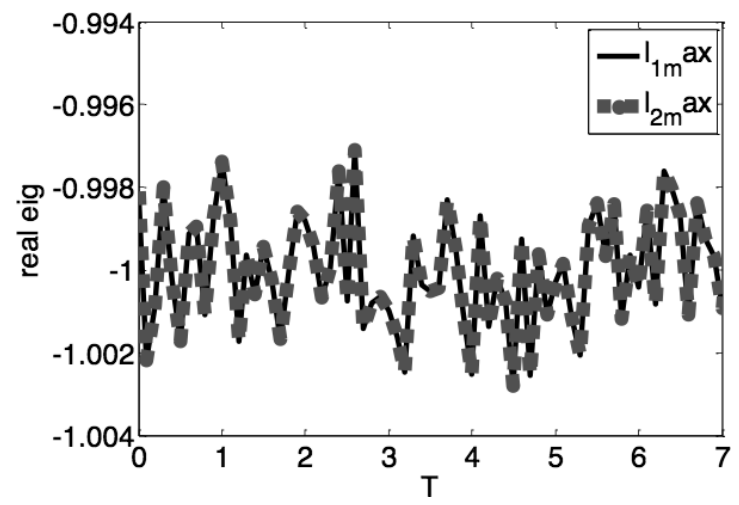

(b) Matrix A(y) (Eq. (45)) eigenvalues

Fig. 7. State observer design with parametric uncertainties.

As it can be seen from Figs 6(a) and (b) the OLFC technique presents better response when the system is not subject to parameter uncertainties and noise measurement. On the other hand, Figs 6(c) and (d) show that the SDRE is more robust when the system is subject to parameter uncertainties and noise measurement.

In Fig. 7 the performance of the state observer design is tested for the same parametric uncertaities, as in the OLFC and SDRE cases. Figure 7(a) shows the error response of the perturbed system. In Fig. 7(b), the eigenvalues of the matrix $\mathbf{A}(\mathbf{y})$ Eq. (45) can be seen, and despite the peturbation over the simulation time, the have negative real parts, assuring stabe error response. Additionally, from Figs 6(a) and (b), it can be seen that the state observer presents the same performance as in the case without parametric uncertainties.

\section{Conclusion}

Three control techniques are used to suppress the chaotic behavior present in a simple MEM resonator, namely: the OLFC, the SDRE and an observer-controller designed with the SDRE. The three techniques proved to be effective in suppressing the chaotic behavior. Additionally, the OLFC, SDRE and state observer methods were tested in the presence of parameter uncertainties and measurement errors, the results pointed out that the SDRE is more robust in this case. Also, the techniques allow the design of the controllers and of the observer without the need of linearization.

\section{Acknowledgments}

The authors would like to thank FAPESP and CNPQ for the financial support, and the organizing committee of the ICNPAA 2012 World Congress: 9th International Conference on Mathematical Problems in Engineering, Aerospace and Science, where part of this work was published.

\section{References}

[1] A.C. Luo and F. Wang, Chaotic motion in a micro-electro-mechanical system with non-linearity from capacitors, Communications in Nonlinear Science and Numerical Simulation 7 (May 2002), 31-49.

[2] S. Liu, A. Davidson and Q. Lin, Simulation studies on nonlinear dynamics and chaos in a mems cantilever control system, Journal of Micromechanics and Microengineering 14(7) (2004), 1064.

[3] M. Ashhab, M.V. Salapaka, M. Dahleh and I. Mezic, Dynamical analysis and control of microcantilevers, Automatica 35(10) (1999), 1663-1670.

[4] A.M. Tusset, A.M. Bueno, C.B. Nascimento, M.D.S. Kaster and J.M. Balthazar, Chaos suppression in nems resonators by using nonlinear control design, AIP Conference Proceedings 1493(1) (2012), 183-189. 
[5] F.R. Chavarette, J.M. Balthazar, J.L. Felix and M. Rafikov, A reducing of a chaotic movement to a periodic orbit, of a micro-electromechanical system, by using an optimal linear control design, Communications in Nonlinear Science and Numerical Simulation 14(5) (2009), 1844-1853.

[6] A.M. Tusset, J.M. Balthazar, D.G. Bassinello, B.R.P. Jr. and J.L.P. Felix, Statements on chaos control designs, including a fractional order dynamical system, applied to a "mems" comb-drive actuator, Nonlinear Dynamics 69(4) (2012), 1837-1857.

[7] A.H. Nayfeh, Perturbation Methods, Pure \& Applied Mathematics, John Wiley \& Sons, 1973.

[8] H.S. Haghighi and A.H. Markazi, Chaos prediction and control in mems resonators, Communications in Nonlinear Science and Numerical Simulation 15(10) (2010), 3091-3099.

[9] H.-T. Yau, C.-C. Wang, C.-T. Hsieh and C.-C. Cho, Nonlinear analysis and control of the uncertain micro-electro-mechanical system by using a fuzzy sliding mode control design, Computers and Mathematics with Applications 61(8) (2011), $1912-1916$.

[10] A. Tusset and J. Balthazar, On the chaotic suppression of both ideal and non-ideal duffing based vibrating systems, using a magnetorheological damper, Differential Equations and Dynamical Systems, 1-17, 10.1007/s12591-012-0128-4.

[11] I. Iliuk, J. Balthazar, A. Tusset, J. Felix and B. de Pontes, On non-ideal and chaotic energy harvester behavior, Differential Equations and Dynamical Systems, pp. 1-12, 10.1007/s12591-012-0127-5.

[12] J.D. Pearson, Approximation methods in optimal control i sub-optimal control, Journal of Electronics and Control 13(5) (1962), $453-469$.

[13] A. Wernli and G. Cook, Suboptimal control for the nonlinear quadratic regulator problem, Automatica 11(1) (1975), 75-84.

[14] C.P. Mracek and J.R. Cloutier, Control designs for the nonlinear benchmark problem via the state-dependent riccati equation method, International Journal of Robust and Nonlinear Control 8(4-5) (1998), 401-433.

[15] B. Friedland, Advanced Control System Design, Prentice-Hall information and system sciences series, Prentice Hall, 1996.

[16] A. Fenili and J.M. Balthazar, The rigid-flexible nonlinear robotic manipulator: Modeling and control, Communications in Nonlinear Science and Numerical Simulation 16(5) (2011), 2332-2341.

[17] M. Rafikov and J.M. Balthazar, On an optimal control design for Rössler system, Physics Letters A 333 (December 2004), $241-245$.

[18] A. Tusset, M. Rafikov and J. Balthazar, An intelligent controller design for magnetorheological damper based on a quarter-car model, Journal of Vibration and Control 15(12) (2009), 1907-1920.

[19] A.M. Tusset, J.M. Balthazar, F.R. Chavarette and J.L.P. Felix, On energy transfer phenomena, in a nonlinear ideal and nonideal essential vibrating systems, coupled to a (mr) magneto-rheological damper, Nonlinear Dynamics 69(4) (2012), 1859-1880.

[20] R. Mestrom, R. Fey, J. van Beek, K. Phan and H. Nijmeijer, Modelling the dynamics of a mems resonator: Simulations and experiments, Sensors and Actuators A: Physical 142(1) (2008), 306-315.

[21] A. Nayfeh and B. Balachandran, Applied Nonlinear Dynamics: Analytical, Computational and Experimental Methods, Wiley Series in Nonlinear Science, John Wiley \& Sons, 2008.

[22] R. Burden and J. Faires, Numerical Analysis, Brooks/Cole, Cengage Learning, 2010.

[23] M. J. Shirazi, R. Vatankhah, M. Boroushaki, H. Salarieh and A. Alasty, Application of particle swarm optimization in chaos synchronization in noisy environment in presence of unknown parameter uncertainty, Communications in Nonlinear Science and Numerical Simulation 17(2) (2012), 742-753.

[24] H.T. Banks, S.C. Beeler and H.T. Tran, State estimation and tracking control of nonlinear dynamical systems, in: Control and Estimation of Distributed Parameter Systems, vol. 143 of International Series of Numerical Mathematics, W. Desch, F. Kappel and K. Kunisch, eds, Birkhäuser Basel, 2003, pp. 1-24.

[25] H. Yabuno, Stabilization and utilization of nonlinear phenomena based on bifurcation control for slow dynamics, Journal of Sound and Vibration 315(3) (2008), 766-780, EUROMECH colloquium 483, Geometrically non-linear vibrations of structures.

[26] S. Rützel, S.I. Lee and A. Raman, Nonlinear dynamics of atomic-force-microscope probes driven in lennard-jones potentials, Proceedings of the Royal Society of London, Series A: Mathematical, Physical and Engineering Sciences 459(2036) (08 2003), $1925-1948$.

[27] J.M. Balthazar, A.M. Tusset, A.M. Bueno and B.R. de Pontes Júnior, On an overview of nonlinear and chaotic behavior and their controls of an atomic force microscopy (afm) vibrating problem, in: Nonlinearity, Bifurcation and Chaos - Theory and Applications, (Vol. 1, chapter 3), J. Awrejcewicz and P. Hagedorn, eds, InTech, 2012, pp. 45-68. 

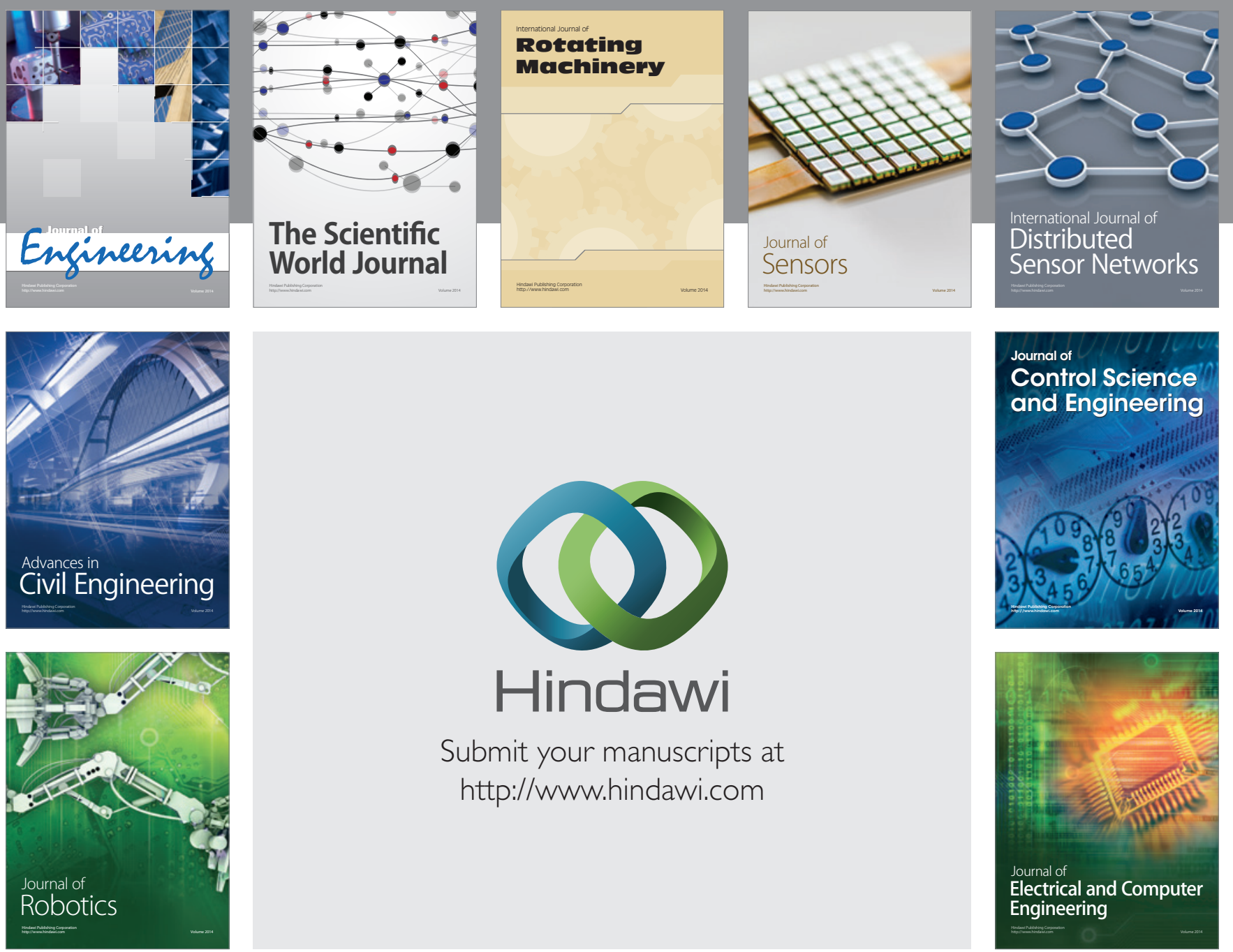

Submit your manuscripts at

http://www.hindawi.com
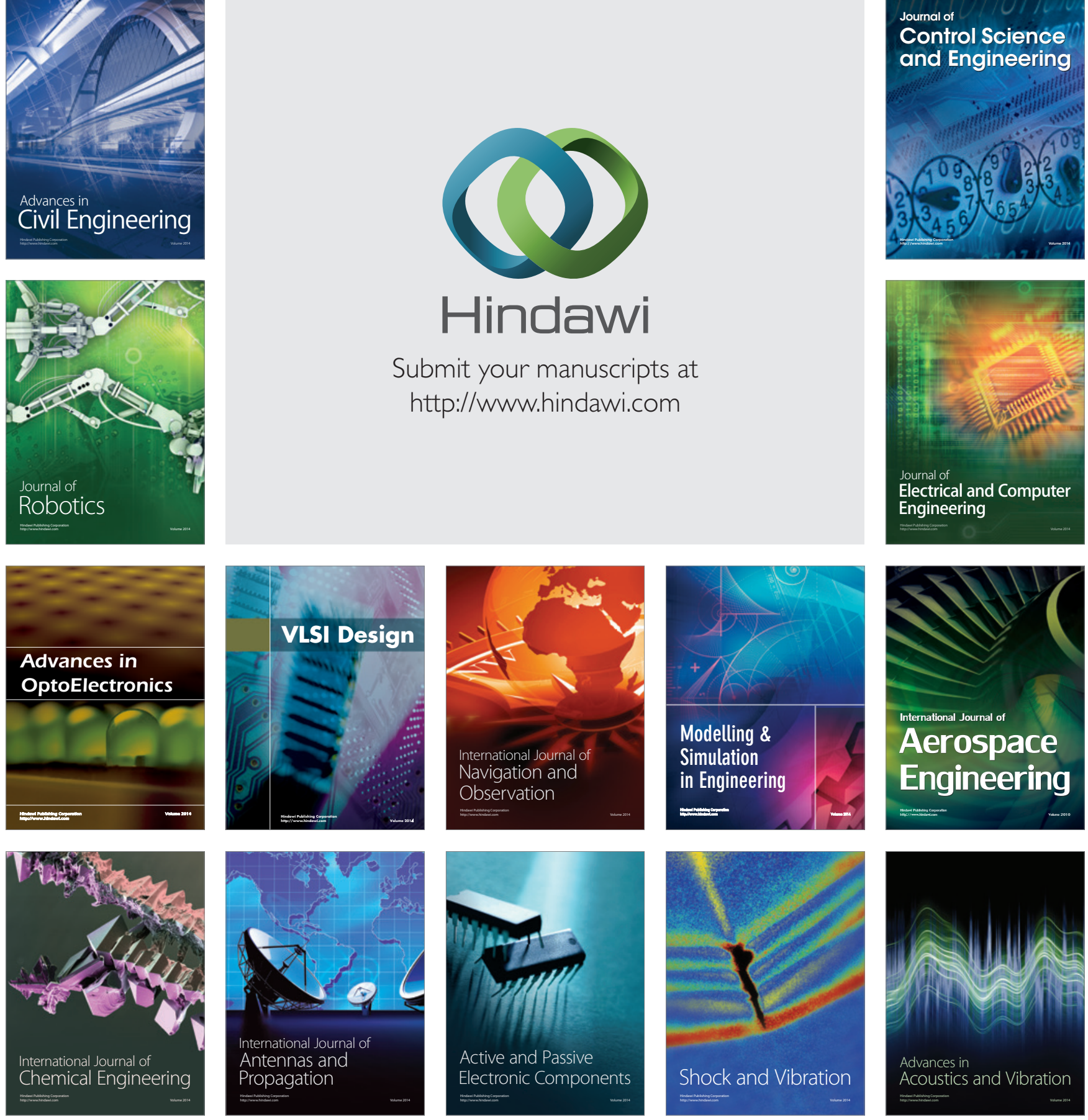